\title{
First Measurement of the $W$-Boson Mass in Run II of the Tevatron
}

T. Aaltonen, ${ }^{23}$ A. Abulencia, ${ }^{24}$ J. Adelman, ${ }^{13}$ T. Affolder, ${ }^{10}$ T. Akimoto, ${ }^{55}$ M. G. Albrow, ${ }^{17}$ S. Amerio, ${ }^{43}$ D. Amidei, ${ }^{35}$ A. Anastassov, ${ }^{52}$ K. Anikeev, ${ }^{17}$ A. Annovi, ${ }^{19}$ J. Antos, ${ }^{14}$ M. Aoki, ${ }^{55}$ G. Apollinari, ${ }^{17}$ T. Arisawa, ${ }^{57}$ A. Artikov, ${ }^{15}$ W. Ashmanskas, ${ }^{17}$ A. Attal, ${ }^{3}$ A. Aurisano, ${ }^{53}$ F. Azfar, ${ }^{42}$ P. Azzi-Bacchetta, ${ }^{43}$ P. Azzurri, ${ }^{46}$ N. Bacchetta, ${ }^{43}$ W. Badgett, ${ }^{17}$ A. Barbaro-Galtieri, ${ }^{29}$ V.E. Barnes, ${ }^{48}$ B. A. Barnett, ${ }^{25}$ S. Baroiant,${ }^{7}$ V. Bartsch, ${ }^{31}$ G. Bauer, ${ }^{33}$ P.-H. Beauchemin, ${ }^{34}$ F. Bedeschi, ${ }^{46}$ S. Behari, ${ }^{25}$ G. Bellettini, ${ }^{46}$ J. Bellinger ${ }^{59}$ A. Belloni, ${ }^{33}$ D. Benjamin,${ }^{16}$ A. Beretvas, ${ }^{17}$ J. Beringer, ${ }^{29}$ T. Berry, ${ }^{30}$ A. Bhatti, ${ }^{50}$ M. Binkley, ${ }^{17}$ D. Bisello, ${ }^{43}$ I. Bizjak, ${ }^{31}$ R. E. Blair, ${ }^{2}$ C. Blocker, ${ }^{6}$ B. Blumenfeld, ${ }^{25}$ A. Bocci, ${ }^{16}$ A. Bodek, ${ }^{49}$ V. Boisvert, ${ }^{49}$ G. Bolla ${ }^{48}$ A. Bolshov, ${ }^{33}$ D. Bortoletto,${ }^{48}$ J. Boudreau ${ }^{47}$ A. Boveia,${ }^{10}$ B. Brau, ${ }^{10}$ L. Brigliadori, ${ }^{5}$ C. Bromberg,${ }^{36}$ E. Brubaker, ${ }^{13}$ J. Budagov, ${ }^{15}$ H. S. Budd,${ }^{49}$ S. Budd,${ }^{24}$ K. Burkett, ${ }^{17}$ G. Busetto, ${ }^{43}$ P. Bussey, ${ }^{21}$ A. Buzatu, ${ }^{34}$ K. L. Byrum, ${ }^{2}$ S. Cabrera, ${ }^{16, q}$ M. Campanelli, ${ }^{20}$ M. Campbell, ${ }^{35}$ F. Canelli, ${ }^{17}$ A. Canepa ${ }^{45}$ S. Carrillo, ${ }^{18, i}$ D. Carlsmith, ${ }^{59}$ R. Carosi, ${ }^{46}$ S. Carron, ${ }^{34}$ B. Casal, ${ }^{11}$ M. Casarsa,${ }^{54}$ A. Castro, ${ }^{5}$ P. Catastini, ${ }^{46}$ D. Cauz, ${ }^{54}$ M. Cavalli-Sforza, ${ }^{3}$ A. Cerri, ${ }^{29}$ L. Cerrito, ${ }^{31, \mathrm{~m}}$ S. H. Chang, ${ }^{28}$ Y. C. Chen, ${ }^{1}$ M. Chertok, ${ }^{7}$ G. Chiarelli, ${ }^{46}$ G. Chlachidze, ${ }^{17}$ F. Chlebana, ${ }^{17}$ I. Cho, ${ }^{28}$ K. Cho, ${ }^{28}$ D. Chokheli, ${ }^{15}$ J.P. Chou ${ }^{22}$ G. Choudalakis, ${ }^{33}$ S. H. Chuang, ${ }^{52}$ K. Chung, ${ }^{12}$ W. H. Chung, ${ }^{59}$ Y. S. Chung, ${ }^{49}$ M. Cilijak, ${ }^{46}$ C. I. Ciobanu, ${ }^{24}$ M. A. Ciocci, ${ }^{46}$ A. Clark, ${ }^{20}$ D. Clark, ${ }^{6}$ M. Coca, ${ }^{16}$ G. Compostella, ${ }^{43}$ M.E. Convery, ${ }^{50}$ J. Conway, ${ }^{7}$ B. Cooper, ${ }^{31}$ K. Copic, ${ }^{35}$ M. Cordelli, ${ }^{19}$ G. Cortiana, ${ }^{43}$ F. Crescioli, ${ }^{46}$ C. Cuenca Almenar, ${ }^{7, q}$ J. Cuevas, ${ }^{11,1}$ R. Culbertson, ${ }^{17}$ J. C. Cully, ${ }^{35}$ S. DaRonco, ${ }^{43}$ M. Datta, ${ }^{17}$ S. D'Auria,${ }^{21}$ T. Davies,${ }^{21}$ D. Dagenhart, ${ }^{17}$ P. de Barbaro, ${ }^{49}$ S. De Cecco, ${ }^{51}$ A. Deisher, ${ }^{29}$ G. De Lentdecker, ${ }^{49, \mathrm{c}}$ G. De Lorenzo, ${ }^{3}$ M. Dell'Orso, ${ }^{46}$ F. Delli Paoli, ${ }^{43}$ L. Demortier, ${ }^{50}$ J. Deng, ${ }^{16}$ M. Deninno, ${ }^{5}$ D. De Pedis,${ }^{51}$ P. F. Derwent, ${ }^{17}$ G. P. Di Giovanni,${ }^{44}$ C. Dionisi,${ }^{51}$ B. Di Ruzza, ${ }^{54}$ J. R. Dittmann, ${ }^{4}$ M. D’Onofrio, ${ }^{3}$ C. Dörr, ${ }^{26}$ S. Donati, ${ }^{46}$ P. Dong, ${ }^{8}$ J. Donini, ${ }^{43}$ T. Dorigo ${ }^{43}$ S. Dube, ${ }^{52}$ J. Efron, ${ }^{39}$ R. Erbacher, ${ }^{7}$ D. Errede, ${ }^{24}$ S. Errede, ${ }^{24}$ R. Eusebi, ${ }^{17}$ H. C. Fang, ${ }^{29}$ S. Farrington,${ }^{30}$ I. Fedorko, ${ }^{46}$ W. T. Fedorko, ${ }^{13}$

R. G. Feild, ${ }^{60}$ M. Feindt, ${ }^{26}$ J. P. Fernandez, ${ }^{32}$ R. Field, ${ }^{18}$ G. Flanagan, ${ }^{48}$ R. Forrest, ${ }^{7}$ S. Forrester, ${ }^{7}$ M. Franklin, ${ }^{22}$

J. C. Freeman, ${ }^{29}$ I. Furic ${ }^{13}$ M. Gallinaro, ${ }^{50}$ J. Galyardt, ${ }^{12}$ J. E. Garcia,${ }^{46}$ F. Garberson, ${ }^{10}$ A. F. Garfinkel,${ }^{48}$ C. Gay, ${ }^{60}$ H. Gerberich ${ }^{24}$ D. Gerdes,${ }^{35}$ S. Giagu, ${ }^{51}$ P. Giannetti ${ }^{46}$ K. Gibson, ${ }^{47}$ J. L. Gimmell, ${ }^{49}$ C. Ginsburg, ${ }^{17}$ N. Giokaris, ${ }^{15, a}$ M. Giordani, ${ }^{54}$ P. Giromini, ${ }^{19}$ M. Giunta, ${ }^{46}$ G. Giurgiu, ${ }^{25}$ V. Glagolev, ${ }^{15}$ D. Glenzinski, ${ }^{17}$ M. Gold, ${ }^{37}$ N. Goldschmidt, ${ }^{18}$ J. Goldstein, ${ }^{42, \mathrm{~b}}$ A. Golossanov, ${ }^{17}$ G. Gomez, ${ }^{11}$ G. Gomez-Ceballos, ${ }^{33}$ M. Goncharov, ${ }^{53}$ O. González, ${ }^{32}$ I. Gorelov, ${ }^{37}$ A. T. Goshaw, ${ }^{16}$ K. Goulianos, ${ }^{50}$ A. Gresele, ${ }^{43}$ S. Grinstein, ${ }^{22}$ C. Grosso-Pilcher, ${ }^{13}$ R. C. Group, ${ }^{17}$ U. Grundler, ${ }^{24}$ J. Guimaraes da Costa, ${ }^{22}$ Z. Gunay-Unalan, ${ }^{36}$ C. Haber, ${ }^{29}$ K. Hahn,${ }^{33}$ S. R. Hahn, ${ }^{17}$ E. Halkiadakis, ${ }^{52}$ A. Hamilton, ${ }^{20}$ B.-Y. Han, ${ }^{49}$ J. Y. Han, ${ }^{49}$ R. Handler, ${ }^{59}$ F. Happacher, ${ }^{19}$ K. Hara, ${ }^{55}$ D. Hare ${ }^{52}$ M. Hare, ${ }^{56}$ S. Harper, ${ }^{42}$ R. F. Harr, ${ }^{58}$ R. M. Harris,${ }^{17}$ M. Hartz,${ }^{47}$ K. Hatakeyama,${ }^{50}$ J. Hauser, ${ }^{8}$ C. Hays,${ }^{42}$ M. Heck,${ }^{26}$ A. Heijboer, ${ }^{45}$ B. Heinemann, ${ }^{29}$ J. Heinrich, ${ }^{45}$ C. Henderson, ${ }^{33}$ M. Herndon, ${ }^{59}$ J. Heuser ${ }^{26}$ D. Hidas, ${ }^{16}$ C. S. Hill, ${ }^{10, b}$ D. Hirschbuehl, ${ }^{26}$ A. Hocker, ${ }^{17}$ A. Holloway, ${ }^{22}$ S. Hou, ${ }^{1}$ M. Houlden, ${ }^{30}$ S.-C. Hsu, ${ }^{9}$ B. T. Huffman, ${ }^{42}$ R. E. Hughes, ${ }^{39}$ U. Husemann, ${ }^{60}$ J. Huston, ${ }^{36}$ J. Incandela, ${ }^{10}$ G. Introzzi, ${ }^{46}$ M. Iori,${ }^{51}$ A. Ivanov, ${ }^{7}$ B. Iyutin,${ }^{33}$ E. James, ${ }^{17}$ D. Jang, ${ }^{52}$ B. Jayatilaka, ${ }^{16}$ D. Jeans,${ }^{51}$ E. J. Jeon, ${ }^{28}$ S. Jindariani, ${ }^{18}$ W. Johnson, ${ }^{7}$ M. Jones, ${ }^{48}$ K. K. Joo,${ }^{28}$ S. Y. Jun, ${ }^{12}$ J. E. Jung, ${ }^{28}$ T. R. Junk ${ }^{24}$ T. Kamon, ${ }^{53}$

P. E. Karchin, ${ }^{58}$ Y. Kato, ${ }^{41}$ Y. Kemp,${ }^{26}$ R. Kephart, ${ }^{17}$ U. Kerzel,,${ }^{26}$ V. Khotilovich, ${ }^{53}$ B. Kilminster, ${ }^{39}$ D. H. Kim, ${ }^{28}$ H. S. Kim, ${ }^{28}$ J.E. Kim, ${ }^{28}$ M. J. Kim, ${ }^{17}$ S. B. Kim,${ }^{28}$ S. H. Kim ${ }^{55}$ Y. K. Kim,,${ }^{13}$ N. Kimura,${ }^{55}$ L. Kirsch,${ }^{6}$ S. Klimenko, ${ }^{18}$ M. Klute, ${ }^{33}$ B. Knuteson, ${ }^{33}$ B. R. Ko,${ }^{16}$ K. Kondo,${ }^{57}$ D. J. Kong, ${ }^{28}$ J. Konigsberg, ${ }^{18}$ A. Korytov, ${ }^{18}$ A. V. Kotwal, ${ }^{16}$ A. C. Kraan, ${ }^{45}$ J. Kraus, ${ }^{24}$ M. Kreps,${ }^{26}$ J. Kroll ${ }^{45}$ N. Krumnack, ${ }^{4}$ M. Kruse,,${ }^{16}$ V. Krutelyov,${ }^{10}$ T. Kubo, ${ }^{55}$ S. E. Kuhlmann, ${ }^{2}$ T. Kuhr, ${ }^{26}$ N. P. Kulkarni, ${ }^{58}$ Y. Kusakabe, ${ }^{57}$ S. Kwang, ${ }^{13}$ A. T. Laasanen, ${ }^{48}$ S. Lai, ${ }^{34}$ S. Lami, ${ }^{46}$ S. Lammel, ${ }^{17}$ M. Lancaster, ${ }^{31}$ R. L. Lander, ${ }^{7}$ K. Lannon, ${ }^{39}$ A. Lath,${ }^{52}$ G. Latino, ${ }^{46}$ I. Lazzizzera, ${ }^{43}$ T. LeCompte, ${ }^{2}$ J. Lee, ${ }^{49}$ J. Lee, ${ }^{28}$ Y. J. Lee, ${ }^{28}$ S. W. Lee, ${ }^{53,0}$ R. Lefèvre, ${ }^{20}$ N. Leonardo, ${ }^{33}$ S. Leone, ${ }^{46}$ S. Levy, ${ }^{13}$ J. D. Lewis, ${ }^{17}$ C. Lin, ${ }^{60}$ C. S. Lin, ${ }^{17}$ M. Lindgren, ${ }^{17}$ E. Lipeles, ${ }^{9}$ T. M. Liss,${ }^{24}$ A. Lister, ${ }^{7}$ D. O. Litvintsev, ${ }^{17}$ T. Liu,,${ }^{17}$ N. S. Lockyer, ${ }^{45}$ A. Loginov, ${ }^{60}$ M. Loreti, ${ }^{43}$ R.-S. Lu, ${ }^{1}$ D. Lucchesi,${ }^{43}$ P. Lujan, ${ }^{29}$ P. Lukens, ${ }^{17}$ G. Lungu, ${ }^{18}$ L. Lyons, ${ }^{42}$ J. Lys,${ }^{29}$ R. Lysak,${ }^{14}$ E. Lytken, ${ }^{48}$ P. Mack, ${ }^{26}$ D. MacQueen, ${ }^{34}$ R. Madrak,${ }^{17}$ K. Maeshima, ${ }^{17}$ K. Makhoul, ${ }^{33}$ T. Maki, ${ }^{23}$ P. Maksimovic,${ }^{25}$ S. Malde, ${ }^{42}$ S. Malik, ${ }^{31}$ G. Manca, ${ }^{30}$ A. Manousakis, ${ }^{15, a}$ F. Margaroli, ${ }^{5}$ R. Marginean,${ }^{17}$ C. Marino, ${ }^{26}$ C. P. Marino, ${ }^{24}$ A. Martin, ${ }^{60}$ M. Martin, ${ }^{25}$ V. Martin, ${ }^{21, \mathrm{~g}}$ M. Martínez, ${ }^{3}$ R. Martínez-Ballarín, ${ }^{32}$ T. Maruyama, ${ }^{55}$ P. Mastrandrea, ${ }^{51}$ T. Masubuchi, ${ }^{55}$

H. Matsunaga, ${ }^{55}$ M. E. Mattson, ${ }^{58}$ R. Mazini, ${ }^{34}$ P. Mazzanti, ${ }^{5}$ K. S. McFarland, ${ }^{49}$ P. McIntyre,${ }^{53}$ R. McNulty, ${ }^{30, f}$ A. Mehta, ${ }^{30}$ P. Mehtala ${ }^{23}$ S. Menzemer,${ }^{11, h}$ A. Menzione, ${ }^{46}$ P. Merkel, ${ }^{48}$ C. Mesropian, ${ }^{50}$ A. Messina, ${ }^{36}$ T. Miao, ${ }^{17}$

N. Miladinovic, ${ }^{6}$ J. Miles, ${ }^{33}$ R. Miller,${ }^{36}$ C. Mills, ${ }^{10}$ M. Milnik, ${ }^{26}$ A. Mitra, ${ }^{1}$ G. Mitselmakher, ${ }^{18}$ A. Miyamoto, ${ }^{27}$ S. Moed,${ }^{20}$ N. Moggi, ${ }^{5}$ B. Mohr, ${ }^{8}$ C. S. Moon,${ }^{28}$ R. Moore, ${ }^{17}$ M. Morello, ${ }^{46}$ P. Movilla Fernandez, ${ }^{29}$ J. Mülmenstädt,${ }^{29}$ 
A. Mukherjee, ${ }^{17}$ Th. Muller ${ }^{26}$ R. Mumford ${ }^{25}$ P. Murat,${ }^{17}$ M. Mussini,${ }^{5}$ J. Nachtman, ${ }^{17}$ A. Nagano, ${ }^{55}$ J. Naganoma, ${ }^{57}$ K. Nakamura, ${ }^{55}$ I. Nakano, ${ }^{40}$ A. Napier, ${ }^{56}$ V. Necula, ${ }^{16}$ C. Neu, ${ }^{45}$ M. S. Neubauer, ${ }^{9}$ J. Nielsen,${ }^{29, \mathrm{n}}$ L. Nodulman, ${ }^{2}$ O. Norniella, ${ }^{3}$ E. Nurse, ${ }^{31}$ S. H. Oh, ${ }^{16}$ Y. D. Oh ${ }^{28}$ I. Oksuzian, ${ }^{18}$ T. Okusawa, ${ }^{41}$ R. Oldeman,${ }^{30}$ R. Orava,${ }^{23}$ K. Osterberg, ${ }^{23}$ C. Pagliarone, ${ }^{46}$ E. Palencia, ${ }^{11}$ V. Papadimitriou, ${ }^{17}$ A. Papaikonomou, ${ }^{26}$ A. A. Paramonov, ${ }^{13}$ B. Parks,${ }^{39}$ S. Pashapour, ${ }^{34}$ J. Patrick, ${ }^{17}$ G. Pauletta, ${ }^{54}$ M. Paulini, ${ }^{12}$ C. Paus,${ }^{33}$ D. E. Pellett, ${ }^{7}$ A. Penzo,${ }^{54}$ T. J. Phillips,${ }^{16}$ G. Piacentino, ${ }^{46}$ J. Piedra, ${ }^{44}$ L. Pinera,${ }^{18}$ K. Pitts, ${ }^{24}$ C. Plager, ${ }^{8}$ L. Pondrom,${ }^{59}$ X. Portell, ${ }^{3}$ O. Poukhov, ${ }^{15}$ N. Pounder,${ }^{42}$ F. Prakoshyn, ${ }^{15}$ A. Pronko, ${ }^{17}$ J. Proudfoot, ${ }^{2}$ F. Ptohos, ${ }^{19, \mathrm{e}}$ G. Punzi, ${ }^{46}$ J. Pursley, ${ }^{25}$ J. Rademacker, ${ }^{42, \mathrm{~b}}$ A. Rahaman, ${ }^{47}$ V. Ramakrishnan, ${ }^{59}$ N. Ranjan, ${ }^{48}$ I. Redondo, ${ }^{32}$ B. Reisert, ${ }^{17}$ V. Rekovic,${ }^{37}$ P. Renton, ${ }^{42}$ M. Rescigno, ${ }^{51}$ S. Richter, ${ }^{26}$ F. Rimondi, ${ }^{5}$ L. Ristori, ${ }^{46}$ A. Robson, ${ }^{21}$ T. Rodrigo, ${ }^{11}$ E. Rogers,${ }^{24}$ S. Rolli, ${ }^{56}$ R. Roser,${ }^{17}$ M. Rossi,${ }^{54}$ R. Rossin,${ }^{10}$ P. Roy,${ }^{34}$ A. Ruiz, ${ }^{11}$ J. Russ, ${ }^{12}$ V. Rusu, ${ }^{13}$ H. Saarikko, ${ }^{23}$ A. Safonov, ${ }^{53}$ W. K. Sakumoto, ${ }^{49}$ G. Salamanna, ${ }^{51}$ O. Saltó, ${ }^{3}$ L. Santi, ${ }^{54}$ S. Sarkar,${ }^{51}$ L. Sartori, ${ }^{46}$ K. Sato, ${ }^{17}$ P. Savard, ${ }^{34}$ A. Savoy-Navarro, ${ }^{44}$ T. Scheidle, ${ }^{26}$ P. Schlabach, ${ }^{17}$ E. E. Schmidt, ${ }^{17}$ M. P. Schmidt, ${ }^{60}$ M. Schmitt, ${ }^{38}$ T. Schwarz, ${ }^{7}$ L. Scodellaro, ${ }^{11}$ A. L. Scott,${ }^{10}$ A. Scribano, ${ }^{46}$ F. Scuri, ${ }^{46}$ A. Sedov, ${ }^{48}$ S. Seidel,${ }^{37}$ Y. Seiya, ${ }^{41}$ A. Semenov, ${ }^{15}$ L. Sexton-Kennedy, ${ }^{17}$ A. Sfyrla, ${ }^{20}$ S.Z. Shalhout,${ }^{58}$ M. D. Shapiro, ${ }^{29}$ T. Shears,${ }^{30}$ P. F. Shepard, ${ }^{47}$ D. Sherman, ${ }^{22}$ M. Shimojima ${ }^{55, k}$ M. Shochet, ${ }^{13}$ Y. Shon, ${ }^{59}$ I. Shreyber, ${ }^{20}$ A. Sidoti, ${ }^{46}$ P. Sinervo, ${ }^{34}$ A. Sisakyan, ${ }^{15}$ A. J. Slaughter, ${ }^{17}$ J. Slaunwhite, ${ }^{39}$ K. Sliwa, ${ }^{56}$ J. R. Smith,${ }^{7}$ F. D. Snider, ${ }^{17}$ R. Snihur,${ }^{34}$ M. Soderberg, ${ }^{35}$ A. Soha, ${ }^{7}$ S. Somalwar, ${ }^{52}$ V. Sorin,${ }^{36}$ J. Spalding, ${ }^{17}$ F. Spinella, ${ }^{46}$ T. Spreitzer,${ }^{34}$ P. Squillacioti, ${ }^{46}$ M. Stanitzki, ${ }^{60}$ A. Staveris-Polykalas, ${ }^{46}$ R. St. Denis, ${ }^{21}$ B. Stelzer, ${ }^{8}$ O. Stelzer-Chilton, ${ }^{42}$ D. Stentz, ${ }^{38}$ J. Strologas,${ }^{37}$ D. Stuart, ${ }^{10}$ J. S. Suh,${ }^{28}$ A. Sukhanov, ${ }^{18}$ H. Sun, ${ }^{56}$ I. Suslov, ${ }^{15}$ T. Suzuki, ${ }^{55}$ A. Taffard, ${ }^{24, p}$ R. Takashima, ${ }^{40}$ Y. Takeuchi, ${ }^{55}$ R. Tanaka, ${ }^{40}$ M. Tecchio, ${ }^{35}$ P. K. Teng, ${ }^{1}$ K. Terashi, ${ }^{50}$ J. Thom, ${ }^{17, \mathrm{~d}}$ A. S. Thompson, ${ }^{21}$ E. Thomson, ${ }^{45}$ P. Tipton, ${ }^{60}$ V. Tiwari, ${ }^{12}$ S. Tkaczyk, ${ }^{17}$ D. Toback, ${ }^{53}$ S. Tokar, ${ }^{14}$ K. Tollefson, ${ }^{36}$ T. Tomura, ${ }^{55}$ D. Tonelli, ${ }^{46}$ S. Torre, ${ }^{19}$ D. Torretta, ${ }^{17} \mathrm{~S}$. Tourneur, ${ }^{44} \mathrm{~W}$. Trischuk, ${ }^{34} \mathrm{~S}$. Tsuno, ${ }^{40} \mathrm{Y}$. Tu, ${ }^{45}$ N. Turini ${ }^{46}$ F. Ukegawa, ${ }^{55}$ S. Uozumi,${ }^{55}$ S. Vallecorsa, ${ }^{20}$ N. van Remortel,${ }^{23}$ A. Varganov, ${ }^{35}$ E. Vataga,${ }^{37}$ F. Vazquez, ${ }^{18, i}$ G. Velev, ${ }^{17}$ C. Vellidis,${ }^{46, a}$ G. Veramendi, ${ }^{24}$ V. Veszpremi, ${ }^{48}$ M. Vidal,${ }^{32}$ R. Vidal,${ }^{17}$ I. Vila, ${ }^{11}$ R. Vilar, ${ }^{11}$ T. Vine, ${ }^{31}$ M. Vogel,${ }^{37}$ I. Vollrath, ${ }^{34}$ I. Volobouev,${ }^{29, o}$ G. Volpi,${ }^{46}$ F. Würthwein,,${ }^{9}$ P. Wagner, ${ }^{53}$ R. G. Wagner, ${ }^{2}$ R. L. Wagner, ${ }^{17}$ J. Wagner, ${ }^{26}$ W. Wagner, ${ }^{26}$ R. Wallny, ${ }^{8}$ S. M. Wang, ${ }^{1}$ A. Warburton, ${ }^{34}$ D. Waters,${ }^{31}$ M. Weinberger, ${ }^{53}$ W. C. Wester III, ${ }^{17}$ B. Whitehouse, ${ }^{56}$ D. Whiteson, ${ }^{45, p}$ A. B. Wicklund,${ }^{2}$ E. Wicklund, ${ }^{17}$ G. Williams, ${ }^{34}$ H. H. Williams, ${ }^{45}$ P. Wilson, ${ }^{17}$ B. L. Winer, ${ }^{39}$ P. Wittich, ${ }^{17, d}$ S. Wolbers, ${ }^{17}$ C. Wolfe, ${ }^{13}$ T. Wright,${ }^{35} \mathrm{X}$. Wu,${ }^{20}$ S. M. Wynne, ${ }^{30}$ A. Yagil, ${ }^{9}$ K. Yamamoto, ${ }^{41}$ J. Yamaoka, ${ }^{52}$ T. Yamashita, ${ }^{40}$ C. Yang,${ }^{60}$ U. K. Yang,,${ }^{13}$ Y. C. Yang, ${ }^{28}$ W. M. Yao, ${ }^{29}$ G. P. Yeh,${ }^{17}$ J. Yoh, ${ }^{17}$ K. Yorita, ${ }^{13}$ T. Yoshida ${ }^{41}$ G. B. Yu, ${ }^{49}$ I. Yu, ${ }^{28}$ S. S. Yu, ${ }^{17}$ J. C. Yun, ${ }^{17}$ L. Zanello, ${ }^{51}$ A. Zanetti, ${ }^{54}$ I. Zaw, ${ }^{22}$ X. Zhang, ${ }^{24}$ J. Zhou, ${ }^{52}$ and S. Zucchelli ${ }^{5}$

\title{
(CDF Collaboration)
}

\author{
${ }^{1}$ Institute of Physics, Academia Sinica, Taipei, Taiwan 11529, Republic of China \\ ${ }^{2}$ Argonne National Laboratory, Argonne, Illinois 60439, USA \\ ${ }^{3}$ Institut de Fisica d'Altes Energies, Universitat Autonoma de Barcelona, E-08193, Bellaterra (Barcelona), Spain \\ ${ }^{4}$ Baylor University, Waco, Texas 76798, USA \\ ${ }^{5}$ Istituto Nazionale di Fisica Nucleare, University of Bologna, I-40127 Bologna, Italy \\ ${ }^{6}$ Brandeis University, Waltham, Massachusetts 02254, USA \\ ${ }^{7}$ University of California, Davis, Davis, California 95616, USA \\ ${ }^{8}$ University of California, Los Angeles, Los Angeles, California 90024, USA \\ ${ }^{9}$ University of California, San Diego, La Jolla, California 92093, USA \\ ${ }^{10}$ University of California, Santa Barbara, Santa Barbara, California 93106, USA \\ ${ }^{11}$ Instituto de Fisica de Cantabria, CSIC-University of Cantabria, 39005 Santander, Spain \\ ${ }^{12}$ Carnegie Mellon University, Pittsburgh, Pennsylvania 15213, USA \\ ${ }^{13}$ Enrico Fermi Institute, University of Chicago, Chicago, Illinois 60637, USA \\ ${ }^{14}$ Comenius University, 84248 Bratislava, Slovakia; and Institute of Experimental Physics, 04001 Kosice, Slovakia \\ ${ }^{15}$ Joint Institute for Nuclear Research, RU-141980 Dubna, Russia \\ ${ }^{16}$ Duke University, Durham, North Carolina 27708, USA \\ ${ }^{17}$ Fermi National Accelerator Laboratory, Batavia, Illinois 60510, USA \\ ${ }^{18}$ University of Florida, Gainesville, Florida 32611, USA \\ ${ }^{19}$ Laboratori Nazionali di Frascati, Istituto Nazionale di Fisica Nucleare, I-00044 Frascati, Italy \\ ${ }^{20}$ University of Geneva, CH-1211 Geneva 4, Switzerland \\ ${ }^{21}$ Glasgow University, Glasgow G12 8QQ, United Kingdom \\ ${ }^{22}$ Harvard University, Cambridge, Massachusetts 02138, USA
}




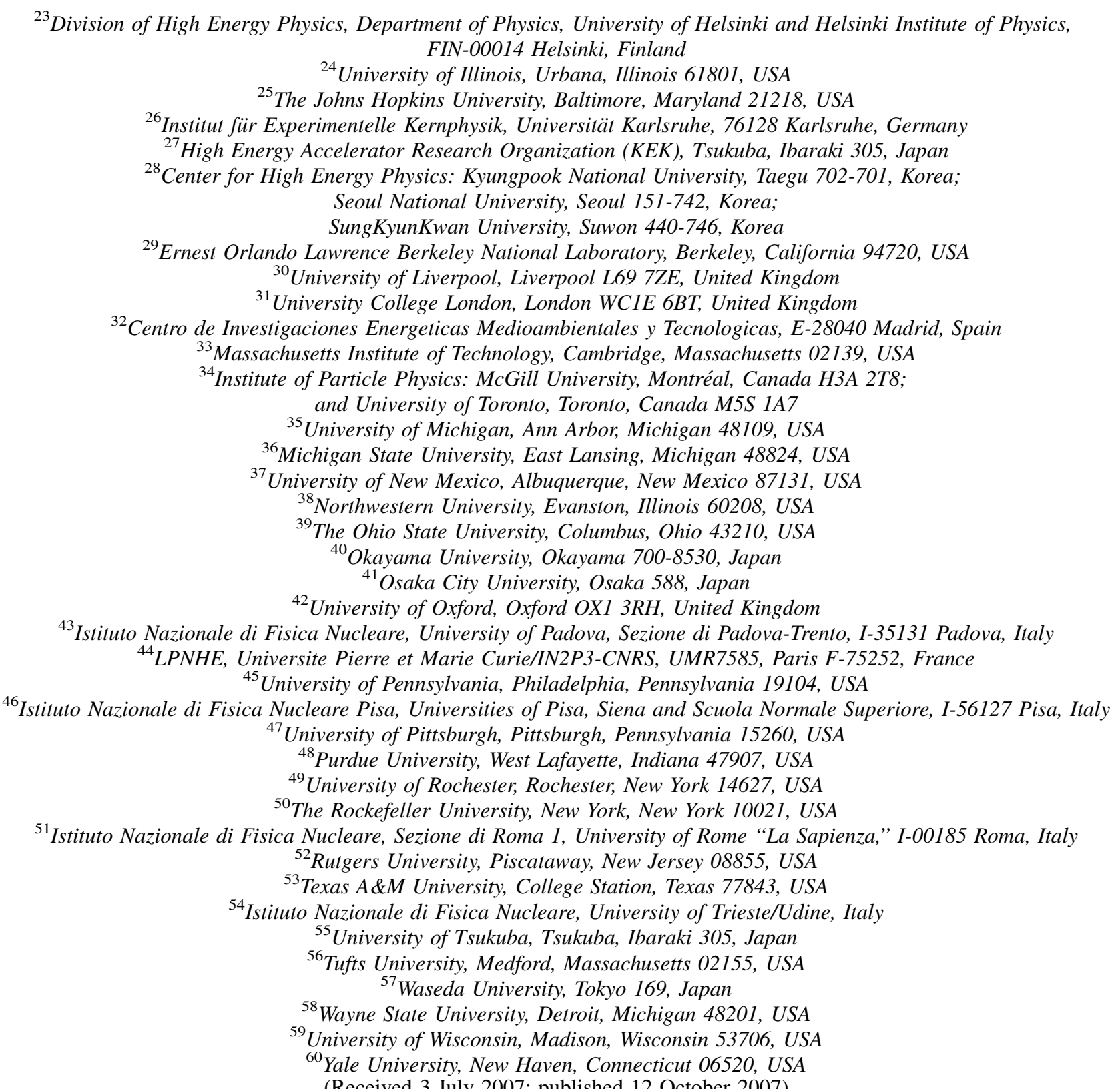

\begin{abstract}
We present a measurement of the $W$-boson mass using $200 \mathrm{pb}^{-1}$ of data collected in $p \bar{p}$ collisions at $\sqrt{s}=1.96 \mathrm{TeV}$ by the CDF II detector at run II of the Fermilab Tevatron. With a sample of $63964 \mathrm{~W} \rightarrow$ $e \nu$ candidates and $51128 \mathrm{~W} \rightarrow \mu \nu$ candidates, we measure $M_{W}=80413 \pm 34_{\text {stat }} \pm 34_{\text {syst }}=80413 \pm$ $48 \mathrm{MeV} / c^{2}$. This is the most precise single measurement of the $W$-boson mass to date.
\end{abstract}

The standard model (SM) invokes the Higgs mechanism of spontaneous symmetry breaking to generate mass for the $W$ and $Z$ bosons, which mediate the weak force. The $S U(2) \times U(1)$ symmetry of the electroweak interaction predicts the relation between the $W$ - and $Z$-boson masses and the electromagnetic and weak gauge couplings. The prediction for the $W$-boson mass $M_{W}$ in terms of the precisely measured $Z$-boson mass $M_{Z}$, the Fermi decay constant $G_{F}$ extracted from the muon lifetime measure- ment, and the electromagnetic coupling $\alpha$ at the scale $M_{Z}$ are given in the "on-shell" scheme by [1]

$$
M_{W}^{2}=\frac{\hbar^{3}}{c} \frac{\pi \alpha}{\sqrt{2} G_{F}} \frac{1}{\left(1-c_{W}^{2}\right)(1-\Delta r)},
$$

where $c_{W}=M_{W} / M_{Z}$ and $\Delta r$ is the quantum-loop correction. A precise measurement of $M_{W}$ provides a measurement of $\Delta r$. In the $\mathrm{SM}$ the contributions to $\Delta r$ are dominated by the top quark and the Higgs boson loops, 
such that $M_{W}$ in conjunction with the top quark mass constrains the mass $m_{H}$ of the undiscovered Higgs boson. An $m_{H}$ constraint, inconsistent with direct searches, can indicate the presence of new physics, such as contributions to $\Delta r$ from supersymmetric particles [2].

The $W$-boson mass [1] has been measured most precisely by the LEP $[3,4]$ and Tevatron [5] experiments, with the world average $M_{W}=80392 \pm 29 \mathrm{MeV} / c^{2}$ [4]. At the Tevatron, $W$ bosons are mainly produced in quark $\left(q^{\prime}\right)$ antiquark $(\bar{q})$ annihilation $q^{\prime} \bar{q} \rightarrow W+X$. Here $X$ includes the QCD radiation that forms the "hadronic recoil" balancing the boson's transverse momentum $p_{T}$ [6]. The $W \rightarrow$ $\ell \nu$ decays, characterized by a high- $p_{T}$ charged lepton $(\ell=$ $e$ or $\mu$ ) and neutrino, can be selected with high purity and provide precise mass information.

This analysis [7,8] uses $200 \mathrm{pb}^{-1}$ collected by the CDF II detector [7] in $p \bar{p}$ collisions at $\sqrt{s}=1.96 \mathrm{TeV}$ at the Tevatron. CDF II is a magnetic spectrometer surrounded by calorimeters and muon detectors. We use the central drift chamber (COT) [9], the central calorimeter [10] with embedded wire chambers [11] at the electromagnetic (EM) shower maximum, and the muon detectors [12] for identification of muons and electrons with $|\eta|<1$ [6] and measurement of their four-momenta. The muon (electron) trigger requires a COT track with $p_{T}>18(9) \mathrm{GeV} / c$ [6] and matching muon chamber hits (EM calorimeter cluster with $E_{T}>18 \mathrm{GeV}$ ).

In the analysis, we select muons with a COT track matched to muon chamber hits and passing quality requirements, track $p_{T}>30 \mathrm{GeV} / c$, and a minimum-ionization signal in the calorimeter. Cosmic rays are rejected using COT hit timing [13]. We select electrons with track $p_{T}>$ $18 \mathrm{GeV} / c$, EM cluster $E_{T}>30 \mathrm{GeV}$ [6,7], and passing quality requirements on the COT track and the trackcluster matching. Additional requirements are based on the ratio of the calorimeter energy $E$ to track momentum $p(E / p c<2)$, the ratio of energies detected in the hadronic and EM calorimeters $E_{\mathrm{Had}} / E_{\mathrm{EM}}<0.1$, and the transverse shower profile [7]. A veto on the presence of a second lepton suppresses $Z$-boson background, with negligible loss of $W$-boson events. Control samples of $Z$-boson events require two oppositely charged leptons with the above criteria.

The $\vec{p}_{T}$ of the hadronic recoil $(\vec{u})$ equals the vector sum $\vec{u}=\Sigma_{i} E_{i} \sin \left(\theta_{i}\right) \hat{n}_{i} / c$ over calorimeter towers [10], with energy $E_{i}$, polar angle $\theta_{i}$, and transverse directions specified by unit vectors $\hat{n}_{i}$. Energy associated with the charged lepton(s) is not included. We impose $\vec{p}_{T}$ balance to infer the neutrino's transverse momentum $p_{T}^{\nu} \equiv\left|-\vec{p}_{T}^{\ell}-\vec{u}\right|[6]$ and the $W$ transverse mass $m_{T}=\sqrt{2\left(p_{T}^{\ell} p_{T}^{\nu}-\vec{p}_{T}^{\ell} \cdot \vec{p}_{T}^{\nu}\right)} / c$. We require $p_{T}^{\nu}>30 \mathrm{GeV} / c$ and $|\vec{u}|<15 \mathrm{GeV} / c$ to obtain a $W$ candidate sample of high purity, whose $m_{T}$ and lepton $p_{T}$ distributions are strongly correlated with $M_{W}$. The sample consists of $63964 \mathrm{~W} \rightarrow e \nu$ and $51128 W \rightarrow \mu \nu$ candidates.
The $W$-boson mass is extracted by performing binned maximum likelihood fits to the distributions of $m_{T}, p_{T}^{\ell}$, and $p_{T}^{\nu}$. We generate 800 templates as functions of $M_{W}$ between $80 \mathrm{GeV} / c^{2}$ and $81 \mathrm{GeV} / c^{2}$ using a custom Monte Carlo (MC) simulation [7] of boson production and decay, and of the detector response to the lepton(s) and hadronic recoil. The custom MC optimizes computing speed and control of systematic uncertainties. The kinematics of $W$ - and $Z$-boson decays are obtained from the RESBOS [14] program. We tune the nonperturbative form factor in RESBOS, which describes the boson $p_{T}$ spectrum at low $p_{T}$, on the dilepton $p_{T}$ distributions in the $Z$-boson data. Single photons (final-state radiation) radiated from the final-state leptons are generated according to the WGRAD program [15]. The final-state radiation photon energies are increased by $10 \%$ (with an absolute uncertainty of 5\%) to account for additional energy loss due to two-photon radiation [16]. WGRAD is also used to estimate the initial-state QED radiation. We use the CTEQ6M [17] set of parton distribution functions and their uncertainties.

The custom MC simulation performs a hit-level simulation of the lepton track. A fine-grained model of passive material properties is used to calculate ionization and radiative energy loss and multiple Coulomb scattering. Bremsstrahlung photons and conversion electrons are generated and propagated to the calorimeter. COT hits are generated according to the resolution $(\approx 150 \mu \mathrm{m})$ and efficiencies measured from muon tracks in $Y, W$, and $Z$-boson decays. A helix fit (with optional beam constraint) is performed to simulate the reconstructed track.

The alignment of the COT is performed using a highpurity sample of high- $p_{T}$ cosmic ray muons. Each muon's complete trajectory is fit to a single helix [13]. The fits determine the relative locations of the sense wires, including gravitational and electrostatic displacements, with a precision of a few microns. We constrain remaining misalignments, which cause a bias in the track curvature, by comparing $\langle E / p c\rangle$ for electrons and positrons.

The tracker momentum scale is measured by template fitting the $J / \psi \rightarrow \mu \mu$ and $\Upsilon \rightarrow \mu \mu$ mass peaks. The $J / \psi$ fits are performed in bins of $\left\langle 1 / p_{T}^{\ell}(\mu)\right\rangle$ to measure any nonlinearity due to mismodeling of the ionization energy loss and other smaller effects, and in bins of $\langle\cot \theta(\mu)\rangle$ to measure the magnetic field nonuniformity. To account for the observed momentum nonlinearity, a downward 6\% correction to the predicted ionization energy loss is applied in the simulation to make the measured $J / \psi$ mass independent of $\left\langle 1 / p_{T}^{\ell}(\mu)\right\rangle$. The calibration derived from the $J / \psi$ and $Y$ data yields $M_{Z}=91184 \pm 43_{\text {stat }} \mathrm{MeV} / c^{2}$ (Fig. 1) from the $Z \rightarrow \mu \mu$ data, consistent with the world average [1,4] of $91188 \pm 2 \mathrm{MeV} / c^{2}$. The systematic uncertainties due to QED radiative corrections and magnetic field nonuniformity dominate the total uncertainty of $0.02 \%$ on the combined momentum scale, derived from the $J / \psi, \Upsilon$, and Z-boson mass fits. 

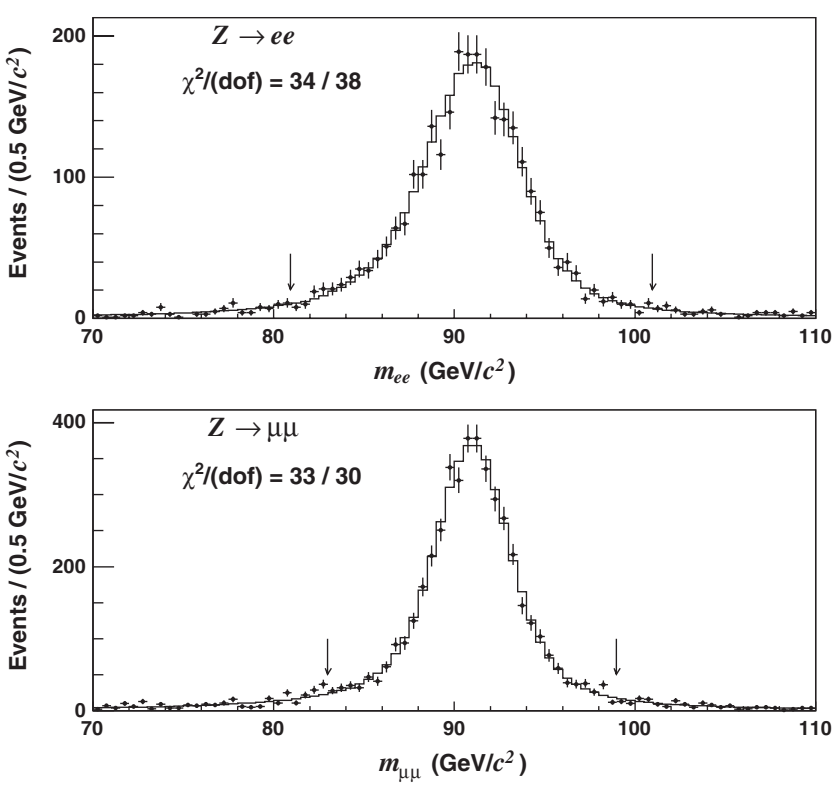

FIG. 1. The $Z \rightarrow e e$ (top panel) and $Z \rightarrow \mu \mu$ (bottom panel) mass fits, showing the data (points) and the simulation (histogram). The arrows indicate the fitting range.

We simulate the electron cluster by merging the energies of the primary electron and the proximate bremsstrahlung photons and conversion electrons. The distributions of electron and photon energy loss in the solenoid coil, and leakage into the hadronic calorimeter, are determined using GEANT [18] as a function of $E_{T}$. The fractional energy resolution is given by the quadrature sum of a sampling term $\left(13.5 \% / \sqrt{E_{T} / \mathrm{GeV}}\right)$ and a constant term $\kappa=(0.89 \pm$ $0.15) \%$ applied to the cluster energy, and an additional constant term $\kappa_{\gamma}=(8.3 \pm 2.2) \%$ applied only to the energies of bremsstrahlung photons and conversion electrons. The $\kappa_{\gamma}$ term contributes $\approx 1.3 \%$ in quadrature to the

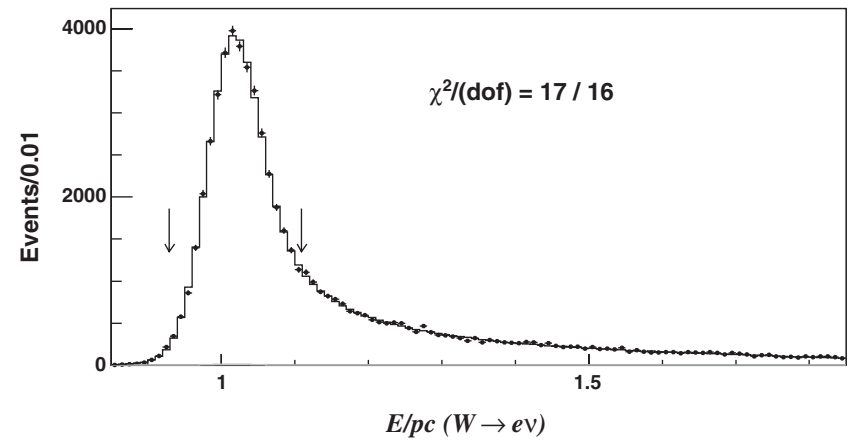

FIG. 2. The distribution of $E / p c$ for the $W \rightarrow e \nu$ data (points) and the best-fit simulation (histogram) including the small jet background (shaded). The arrows indicate the fitting range used for the electron energy calibration. The jet background, which is barely visible on this scale, contributes a negligible uncertainty in the calibrations of the electron energy scale and the amount of radiative material.
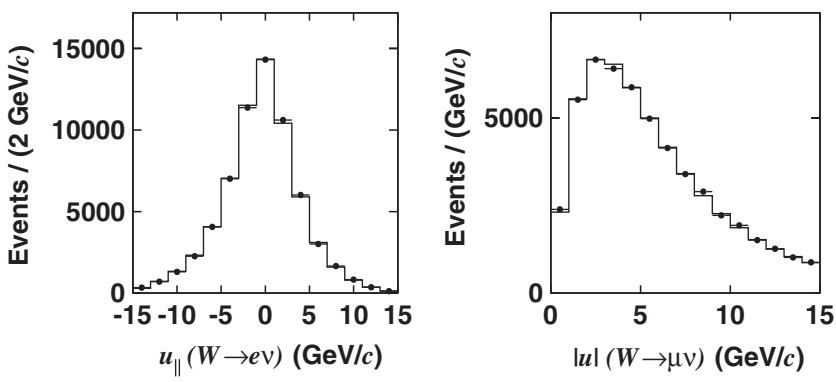

FIG. 3. Left panel: The $u_{\|}$distribution for the electron channel data (points) and simulation (histogram). Right panel: The $|\vec{u}|$ distribution for the muon channel. The mean and rms of the histograms agree between data and simulation, within the statistical precisions of $\approx 1 \%$.

effective constant term for the inclusive electron sample. The distribution of the underlying event energy [7] in the cluster is simulated. We tune $\kappa$ on the width of the $E / p c$ peak (Fig. 2) of the $W$-boson sample, and $\kappa_{\gamma}$ on the width of the $Z \rightarrow e e$ mass peak when both electrons are radiative $(E / p c>1.06)$.

Given the tracker momentum calibration, we fit the $E / p c$ peak in bins of electron $E_{T}$ to determine the electron energy scale and nonlinearity. The position of the $E / p c$ peak is sensitive to the number of radiation lengths $X_{0}(\approx 19 \%)$, due to bremsstrahlung upstream of the COT. We constrain $X_{0}$ by comparing the fraction of electrons with high $E / p c$ between data and simulation. Applying the $E / p c$-based energy calibration, we fit the $Z \rightarrow e e$ mass peak and measure $M_{Z}=91190 \pm 67_{\text {stat }} \mathrm{MeV} / c^{2}$ (Fig. 1), consistent with the world average [1,4]. For maximum precision, the energy scales from the $W E / p c$ fit and the $Z \rightarrow e e$ mass fit are combined using the best linear unbiased estimate (BLUE) method [19], with a resulting uncertainty that is mostly statistical.

The recoil $\vec{u}$ excludes towers in which the lepton(s) deposit energy. The underlying event energy in these towers is measured from the nearby towers in $W$-boson data, including its dependence on $\eta^{\ell}$ and $\vec{u}$. The resolution of $\vec{u}$ has jetlike and underlying event components, with the

TABLE I. Fit results and uncertainties for $M_{W}$. The fit windows are $65-90 \mathrm{GeV} / c^{2}$ for the $m_{T}$ fit and $32-48 \mathrm{GeV} / c$ for the $p_{T}^{\ell}$ and $p_{T}^{\nu}$ fits. The $\chi^{2}$ of the fit is computed using the expected statistical errors on the data points.

\begin{tabular}{lcc}
\hline \hline Distribution & $W$-boson mass $\left(\mathrm{MeV} / c^{2}\right)$ & $\chi^{2} /(\mathrm{dof})$ \\
\hline$m_{T}(e, \nu)$ & $80493 \pm 48_{\text {stat }} \pm 39_{\text {syst }}$ & $86 / 48$ \\
$p_{T}^{\ell}(e)$ & $80451 \pm 58_{\text {stat }} \pm 45_{\text {syst }}$ & $63 / 62$ \\
$p_{T}^{\nu}(e)$ & $80473 \pm 57_{\text {stat }} \pm 54_{\text {syst }}$ & $63 / 62$ \\
$m_{T}(\mu, \nu)$ & $80349 \pm 54_{\text {stat }} \pm 27_{\text {syst }}$ & $59 / 48$ \\
$p_{T}^{\ell}(\mu)$ & $80321 \pm 66_{\text {stat }} \pm 40_{\text {syst }}$ & $72 / 62$ \\
$p_{T}^{\nu}(\mu)$ & $80396 \pm 66_{\text {stat }} \pm 46_{\text {syst }}$ & $44 / 62$ \\
\hline \hline
\end{tabular}



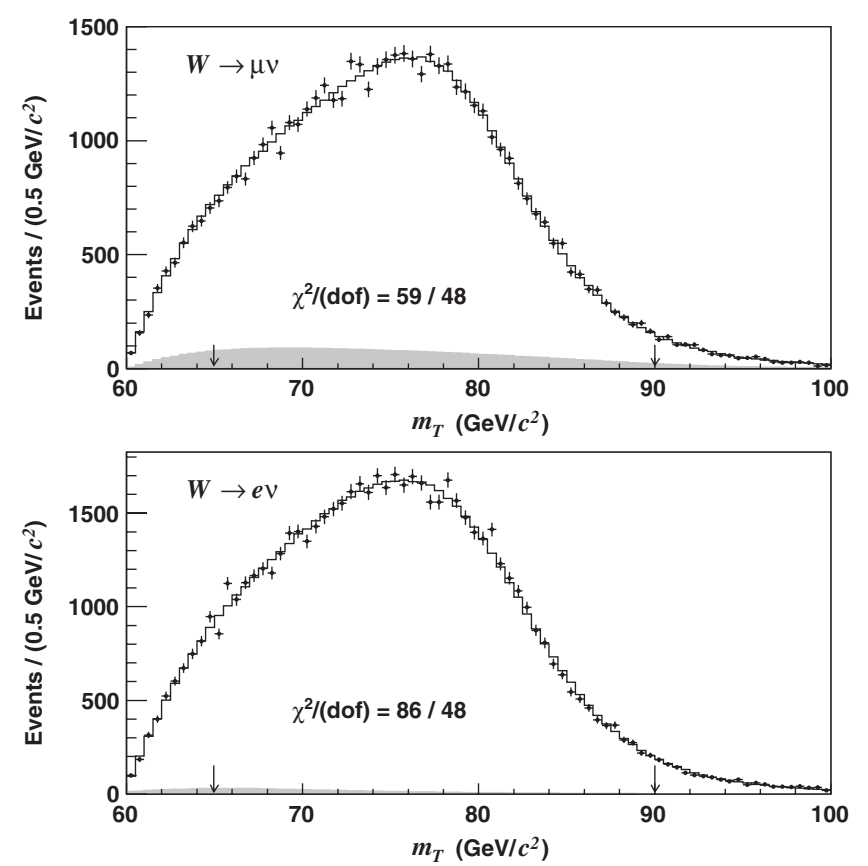

FIG. 4. The $m_{T}$ distribution of the data (points) and the best-fit simulation template (histogram) including backgrounds (shaded), for muons (top panel) and electrons (bottom panel). The arrows indicate the fitting range. The $\chi^{2} /$ (dof) for the electron channel distribution receives large contributions from a few bins near $65 \mathrm{GeV} / c^{2}$, which do not bias the mass fit.

latter modeled using data triggered on inelastic $\bar{p} p$ interactions. The recoil parametrizations are tuned on the mean and rms of the $\vec{p}_{T}$ imbalance between the dilepton $\vec{p}_{T}$ and $\vec{u}$ in $Z \rightarrow \ell \ell$ events. The lepton identification efficiency is measured as a function of $u_{\|}=\vec{u} \cdot \vec{p}_{T}^{\ell} / p_{T}^{\ell}$ using the $Z \rightarrow$ $\ell \ell$ data, in order to model its effect on the $p_{T}^{\ell}$ and $p_{T}^{\nu}$ distributions. Cross-checks of the recoil model using the $W$-boson data show good agreement (Fig. 3).

Backgrounds in the $W$-boson candidate samples arise from misidentified jets containing high- $p_{T}$ tracks and EM clusters, $Z \rightarrow \ell \ell$ where a lepton is not reconstructed and mimics a neutrino, $W \rightarrow \tau \nu, \pi / K$ decays in flight (DIF), and cosmic rays. Jet, DIF, and cosmic ray backgrounds are estimated from the data to be less than $0.5 \%$ combined. The $W \rightarrow \tau \nu$ background is $0.9 \%(0.9 \%)$, and the $Z \rightarrow \ell \ell$ background is $6.6 \%(0.24 \%)$ in the muon (electron) channel, as estimated using a detailed GEANT-based detector simulation. The background shapes are obtained using simulation and data-derived distributions.

Table I shows the fit results from the $m_{T}$ (Fig. 4), $p_{T}^{\ell}$, and $p_{T}^{\nu}$ distributions. These fits are partially uncorrelated and have different systematic uncertainties, thus providing an important cross-check. The fit values were hidden during analysis by adding an unknown offset in the range $[-100,100] \mathrm{MeV} / \mathrm{c}^{2}$. The systematic uncertainties (Table II) were evaluated by fitting MC events to propagate the analysis parameter uncertainties to $M_{W}$.
TABLE II. Systematic and total uncertainties in $\mathrm{MeV} / c^{2}$ for the $m_{T}$ fits, which are the most precise. The last column shows the correlated uncertainties.

\begin{tabular}{lccc}
\hline \hline \multicolumn{1}{c}{ Systematic } & $W \rightarrow e \nu$ & $W \rightarrow \mu \nu$ & Common \\
\hline$p_{T}(W)$ model & 3 & 3 & 3 \\
QED radiation & 11 & 12 & 11 \\
Parton distributions & 11 & 11 & 11 \\
Lepton energy scale & 30 & 17 & 17 \\
Lepton energy resolution & 9 & 3 & 0 \\
Recoil energy scale & 9 & 9 & 9 \\
Recoil energy resolution & 7 & 7 & 7 \\
$u_{\|}$efficiency & 3 & 1 & 0 \\
Lepton removal & 8 & 5 & 5 \\
Backgrounds & 8 & 9 & 0 \\
Total systematic & 39 & 27 & 26 \\
Total uncertainty & 62 & 60 & 26 \\
\hline \hline
\end{tabular}

The consistency of the fit results (Table I) obtained from the different distributions shows that the $W$-boson production, decay, and the hadronic recoil are well modeled. The statistical correlations (evaluated using ensembles of MC events) between the $m_{T}$ and $p_{T}^{\ell}\left(p_{T}^{\nu}\right)$ fit values is $69 \%$ $(68 \%)$, and between the $p_{T}^{\ell}$ and $p_{T}^{\nu}$ fit values is $27 \%$. We numerically combine (using the BLUE method) the six individually fitted $M_{W}$ values, including their correlations, to obtain $M_{W}=80413 \pm 34_{\text {stat }} \pm 34_{\text {syst }} \mathrm{MeV} / \mathrm{c}^{2}$, with $\chi^{2} /($ dof $)=4.8 / 5$. The $m_{T}, p_{T}^{\ell}$, and $p_{T}^{\nu}$ fits in the electron (muon) channel contribute weights of $32.3 \%$ (47.7\%), $8.9 \%(3.4 \%)$, and $6.8 \%(0.9 \%)$, respectively. This establishes an a priori procedure to incorporate the information from individual fits. The muon (electron) channel alone yields $\quad M_{W}=80352 \pm 60 \mathrm{MeV} / c^{2} \quad\left(M_{W}=80477 \pm\right.$ $\left.62 \mathrm{MeV} / c^{2}\right)$ with $\chi^{2} /(\mathrm{dof})=1.4 / 2(0.8 / 2)$. The $m_{T}\left(p_{T}^{\ell}\right.$, $p_{T}^{\nu}$ ) fit results from the muon, and electron channels are consistent with a probability of $7 \%(18 \%, 43 \%)$, taking into account their correlations.

In conclusion, we report the first measurement of the $W$-boson mass from run II of the Tevatron. We measure $M_{W}=80413 \pm 48 \mathrm{MeV} / c^{2}$, the most precise single measurement to date, and we update the world average [4] to $M_{W}=80398 \pm 25 \mathrm{MeV} / c^{2}$. This analysis significantly improves in precision over previous Tevatron measurements, not only through the increased integrated luminosity but also through improved analysis techniques and understanding of systematic uncertainties. As many simulation parameters are constrained by data control samples, their uncertainties are statistical in nature and are expected to be reduced with more data. Inclusion of our result in the global electroweak fit $[4,7]$ reduces the predicted mass of the SM Higgs boson by $6 \mathrm{GeV} / c^{2}$ and decreases its range to $m_{H}=76_{-24}^{+33} \mathrm{GeV} / c^{2}$.

We thank the Fermilab staff and the technical staffs of the participating institutions for their vital contributions. This work was supported by the U.S. Department of 
Energy and National Science Foundation; the Italian Istituto Nazionale di Fisica Nucleare; the Ministry of Education, Culture, Sports, Science and Technology of Japan; the Natural Sciences and Engineering Research Council of Canada; the National Science Council of the Republic of China; the Swiss National Science Foundation; the A.P. Sloan Foundation; the Bundesministerium für Bildung und Forschung, Germany; the Korean Science and Engineering Foundation and the Korean Research Foundation; the Science and Technology Facilities Council and the Royal Society, U.K.; the Institut National de Physique Nucleaire et Physique des Particules/CNRS; the Russian Foundation for Basic Research; the Comisión Interministerial de Ciencia y Tecnología, Spain; the European Community's Human Potential Programme; the European Commission under the Marie Curie Programme; the Slovak R\&D Agency; and the Academy of Finland.

${ }^{\mathrm{a}}$ Visiting scientist from University of Athens, 15784 Athens, Greece.

${ }^{\mathrm{b}}$ Visiting scientist from University of Bristol, Bristol BS8 1TL, United Kingdom.

${ }^{\mathrm{c}}$ Visiting scientist from University Libre de Bruxelles, B-1050 Brussels, Belgium.

${ }^{\mathrm{d}}$ Visiting scientist from Cornell University, Ithaca, NY 14853, USA.

${ }^{\mathrm{e}}$ Visiting scientist from University of Cyprus, Nicosia CY1678 , Cyprus.

${ }^{\mathrm{f}}$ Visiting scientist from University College Dublin, Dublin 4, Ireland.

${ }^{g}$ Visiting scientist from University of Edinburgh, Edinburgh EH9 3JZ, United Kingdom.

${ }^{\mathrm{h}}$ Visiting scientist from University of Heidelberg, D-69120 Heidelberg, Germany.

${ }^{\mathrm{i} V}$ Visiting scientist from Universidad Iberoamericana, Ciudad de México, Mexico.

${ }^{\mathrm{j} V i s i t i n g}$ scientist from University of Manchester, Manchester M13 9PL, United Kingdom.

${ }^{\mathrm{k}}$ Visiting scientist from Nagasaki Institute of Applied Science, Nagasaki, Japan.

${ }^{1}$ Visiting scientist from University de Oviedo, E-33007 Oviedo, Spain.

${ }^{\mathrm{m}}$ Visiting scientist from Queen Mary College, University of London, London E1 4NS, United Kingdom.

${ }^{\mathrm{n}}$ Visiting scientist from University of California, Santa Cruz, Santa Cruz, CA 95064, USA.

${ }^{\circ}$ Visiting scientist from Texas Tech University, Lubbock, TX 79409, USA.
${ }^{\mathrm{p}}$ Visiting scientist from University of California, Irvine, Irvine, CA 92697, USA.

${ }^{\mathrm{q} V}$ Visiting scientist from IFIC (CSIC-Universitat de Valencia), 46071 Valencia, Spain.

[1] W.-M. Yao et al., J. Phys. G 33, 1 (2006).

[2] S. Heinemeyer et al., J. High Energy Phys. 08 (2006) 052.

[3] S. Schael et al. (ALEPH Collaboration), Eur. Phys. J. C 47, 309 (2006); G. Abbiendi et al. (OPAL Collaboration), Eur. Phys. J. C 45, 307 (2006); P. Achard et al. (L3 Collaboration), Eur. Phys. J. C 45, 569 (2006); J. Abdallah et al. (DELPHI Collaboration), Eur. Phys. J. C (to be published).

[4] J. Alcaraz et al. (LEP Collaborations and LEP Electroweak Working Group), CERN Report No. CERN-PH-EP/2006-042, and references therein.

[5] T. Affolder et al. (CDF Collaboration), Phys. Rev. D 64, 052001 (2001); V. M. Abazov et al. (D0 Collaboration), Phys. Rev. D 66, 012001 (2002); B. Abbott et al. (D0 Collaboration), Phys. Rev. D 62, 092006 (2000); B. Abbott et al. (D0 Collaboration), Phys. Rev. D 58, 092003 (1998); V.M. Abazov et al. (CDF and D0 Collaborations), Phys. Rev. D 70, 092008 (2004).

[6] Pseudorapidity is defined as $\eta=-\ln [\tan (\theta / 2)]$, where $\theta$ is the polar angle from the beam axis. Energy (momentum) transverse to the beam is denoted as $E_{T}\left(p_{T}\right)$.

[7] T. Aaltonen et al. (CDF Collaboration), arXiv:0708.3642 [Phys. Rev. D (to be published)].

[8] O. Stelzer-Chilton, Ph.D. thesis, University of Toronto, 2005; I. Vollrath, Ph.D. thesis, University of Toronto, 2006.

[9] T. Affolder et al., Nucl. Instrum. Methods Phys. Res., Sect. A 526, 249 (2004).

[10] F. Abe et al. (CDF Collaboration), Nucl. Instrum. Methods Phys. Res., Sect. A 271, 387 (1988).

[11] A. Byon-Wagner et al., IEEE Trans. Nucl. Sci. 49, 2567 (2002).

[12] G. Ascoli et al., Nucl. Instrum. Methods Phys. Res., Sect. A 268, 33 (1988).

[13] A. V. Kotwal, H.K. Gerberich, and C. Hays, Nucl. Instrum. Methods Phys. Res., Sect. A 506, 110 (2003).

[14] C. Balazs and C.-P. Yuan, Phys. Rev. D 56, 5558 (1997); G. A. Ladinsky and C.-P. Yuan, Phys. Rev. D 50, R4239 (1994); F. Landry, R. Brock, P. M. Nadolsky, and C.-P. Yuan, Phys. Rev. D 67, 073016 (2003).

[15] U. Baur, S. Keller, and D. Wackeroth, Phys. Rev. D 59, 013002 (1998).

[16] C. M. Carloni Calame, G. Montagna, O. Nicrosini, and M. Treccani, Phys. Rev. D 69, 037301 (2004).

[17] J. Pumplin et al., J. High Energy Phys. 07 (2002) 012.

[18] R. Brun and F. Carminati, CERN Program Library Long Writeup, Report No. W5013, 1993 (unpublished), version 3.15 .

[19] L. Lyons, D. Gibaut, and P. Clifford, Nucl. Instrum. Methods Phys. Res., Sect. A 270, 110 (1988). 